\title{
SOME REMARKS ON NON-SYMMETRIC POLARIZATION
}

\author{
FELIPE MARCECA
}

Abstract. Let $P: \mathbb{C}^{n} \rightarrow \mathbb{C}$ be an $m$-homogeneous polynomial given by

$$
P(x)=\sum_{1 \leq j_{1} \leq \ldots \leq j_{m} \leq n} c_{j_{1} \ldots j_{m}} x_{j_{1}} \ldots x_{j_{m}} .
$$

Defant and Schlüters defined a non-symmetric associated $m$-form $L_{P}:\left(\mathbb{C}^{n}\right)^{m} \rightarrow \mathbb{C}$ by

$$
L_{P}\left(x^{(1)}, \ldots, x^{(m)}\right)=\sum_{1 \leq j_{1} \leq \ldots \leq j_{m} \leq n} c_{j_{1} \ldots j_{m}} x_{j_{1}}^{(1)} \ldots x_{j_{m}}^{(m)} .
$$

They estimated the norm of $L_{P}$ on $\left(\mathbb{C}^{n},\|\cdot\|\right)^{m}$ by the norm of $P$ on $\left(\mathbb{C}^{n},\|\cdot\|\right)$ times a $(c \log n)^{m^{2}}$ factor for every 1 -unconditional norm $\|\cdot\|$ on $\mathbb{C}^{n}$. A symmetrization procedure based on a card-shuffling algorithm which (together with Defant and Schlüters' argument) brings the constant term down to $(\mathrm{cm} \log n)^{m-1}$ is provided. Regarding the lower bound, it is shown that the optimal constant is bigger than $(c \log n)^{m / 2}$ when $n \gg m$. Finally, the case of $\ell_{p}$-norms $\|\cdot\|_{p}$ with $1 \leq p<2$ is addressed.

\section{INTRODUCTION}

Let $P: \mathbb{C}^{n} \rightarrow \mathbb{C}$ be an $m$-homogeneous polynomial. It is well-known that there is a unique symmetric $m$-linear form $B:\left(\mathbb{C}^{n}\right)^{m} \rightarrow \mathbb{C}$, such that $B(x, \ldots, x)=P(x)$ for all $x \in \mathbb{C}$. Moreover, the polarization formula gives an expression for the $m$-linear form $B$ in terms of $P$ (see e.g. [3, Section 1.1]). In fact, for every $x^{(1)}, \ldots, x^{(m)} \in \mathbb{C}$, we have

$$
B\left(x^{(1)}, \ldots, x^{(m)}\right)=\frac{1}{2^{m} m !} \sum_{\varepsilon \in\{-1,1\}^{m}} P\left(\varepsilon_{1} x^{(1)}+\ldots+\varepsilon_{m} x^{(m)}\right) .
$$

It follows from this identity that

$$
\sup _{\left\|x^{(k)}\right\| \leq 1}\left|B\left(x^{(1)}, \ldots, x^{(m)}\right)\right| \leq e^{m} \sup _{\|x\| \leq 1}|P(x)|,
$$

for any norm $\|\cdot\|$ in $\mathbb{C}^{n}$.

In [2], Defant and Schlüters defined a non-symmetric $m$-linear form $L_{P}$ arising from a given $m$-homogeneous polynomial $P$. More precisely, for an $m$-homogeneous polynomial $P: \mathbb{C}^{n} \rightarrow \mathbb{C}$ defined by

$$
P(x)=\sum_{1 \leq j_{1} \leq \ldots \leq j_{m} \leq n} c_{j_{1} \ldots j_{m}} x_{j_{1}} \ldots x_{j_{m}}
$$

its associated $m$-linear form $L_{P}:\left(\mathbb{C}^{n}\right)^{m} \rightarrow \mathbb{C}$ is given by

$$
L_{P}\left(x^{(1)}, \ldots, x^{(m)}\right)=\sum_{1 \leq j_{1} \leq \ldots \leq j_{m} \leq n} c_{j_{1} \ldots j_{m}} x_{j_{1}}^{(1)} \ldots x_{j_{m}}^{(m)} .
$$

This work has been supported by CONICET-PIP 11220130100329CO, ANPCyT PICT 2015-2299, UBACyT 20020130100474BA and a CONICET doctoral fellowship. 
Assuming unconditionality of the norm $\|\cdot\|$ in $\mathbb{C}^{n}$, Defant and Schlüters proved that a similar estimate as in (1) holds for $L_{P}$. Before providing further details we introduce an ad hoc definition:

Definition 1.1. For $m, n \in \mathbb{N}$, we define $C(m, n)$ as the infimum of the constants $C>0$ such that for every $m$-homogeneous polynomial $P: \mathbb{C}^{n} \rightarrow \mathbb{C}$ and every 1 unconditional norm $\|\cdot\|$ on $\mathbb{C}^{n}$ we have

$$
\sup _{\left\|x^{(k)}\right\| \leq 1}\left|L_{P}\left(x^{(1)}, \ldots, x^{(m)}\right)\right| \leq C \sup _{\|x\| \leq 1}|P(x)| .
$$

Similarly, for $1 \leq p<2$, we take $C_{p}(m, n)$ as the infimum of the constants $C>0$ such that for every $m$-homogeneous polynomial $P: \mathbb{C}^{n} \rightarrow \mathbb{C}$ we have

$$
\sup _{\left\|x^{(k)}\right\|_{p} \leq 1}\left|L_{P}\left(x^{(1)}, \ldots, x^{(m)}\right)\right| \leq C \sup _{\|x\|_{p} \leq 1}|P(x)| .
$$

The aforementioned result of [2] can be stated in terms of the previous definition.

Theorem 1.2 [2, Theorem 1.1]. There exists a universal constant $c_{1} \geq 1$ such that

$$
C(m, n) \leq\left(c_{1} \log n\right)^{m^{2}} .
$$

Moreover, for $1 \leq p<2$, there is a constant $c_{2}=c_{2}(p) \geq 1$ for which

$$
C_{p}(m, n) \leq c_{2}^{m^{2}}
$$

Note that by the uniqueness of the symmetric $m$-linear form $B$ we have

$$
B\left(x^{(1)}, \ldots, x^{(m)}\right)=\frac{1}{m !} \sum_{\sigma \in \Sigma_{m}} L_{P}\left(x^{\sigma(1)}, \ldots, x^{\sigma(m)}\right),
$$

where $\Sigma_{m}$ is the group of permutations of $m$ elements. The proof of Theorem 1.2 consists of bounding the norm of $L_{P}$ by successive partial symmetrizations starting at $L_{P}$ and ending at the fully symmetrized $B$. Finally, applying (1) yields the result. Changing only the way in which this symmetrization is carried out and using the same arguments as in [2], we obtain improved bounds for the constants $C(m, n)$ and $C_{p}(m, n)$. Additionally, we provide lower bounds for these constants. Our main result is the following.

Theorem 1.3. There exists a universal constant $c_{1} \geq 1$ such that

$$
\left(\frac{\log \left(\frac{2 n}{m}\right)-\pi}{\pi}\right)^{m / 2} \leq C(m, n) \leq c_{1}^{m} m^{m}(\log n)^{m-1} .
$$

Moreover, for $1 \leq p<2$, there is a constant $c_{2}=c_{2}(p) \geq 1$ for which

$$
m^{\frac{m}{p}} \leq C_{p}(m, n) \leq c_{2}^{m} m^{m} .
$$

Remark 1.4. Defant and Schlüters achieved similar upper bounds by refining their original calculations from [2] as it was mentioned during a personal communication.

Remark 1.5. Scrutiny of the theorem's proof suggests that the underlying reason which determines the magnitude of the constants $C(m, n)$ and $C_{p}(m, n)$ is the behaviour of the operator known as the main triangle projection. Roughly speaking, the main triangle projection is the operator which given a matrix in $\mathbb{C}^{n \times n}$ returns the same matrix with zeroes below the diagonal. Each norm on $\mathbb{C}^{n}$ induces an operator norm in $\mathbb{C}^{n \times n}$ and again this induces a norm for the main triangle projection. Estimations 
of the latter norm are the ones that shape the upper and lower bounds of $C(m, n)$ and $C_{p}(m, n)$ that were obtained.

\section{SyMmetrizATION}

The following may be deduced from (2).

$$
\begin{aligned}
B\left(x^{(1)}, \ldots, x^{(m)}\right) & =\frac{1}{m !} \sum_{\sigma \in \Sigma_{m}} L_{P}\left(x^{\sigma(1)}, \ldots, x^{\sigma(m)}\right) \\
& =\frac{1}{m !} \sum_{\sigma \in \Sigma_{m}} \sum_{1 \leq j_{1} \leq \ldots \leq j_{m} \leq n} c_{j_{1} \ldots j_{m}} x_{j_{1}}^{\sigma(1)} \ldots x_{j_{m}}^{\sigma(m)} \\
& =\frac{1}{m !} \sum_{\sigma \in \Sigma_{m}} \sum_{1 \leq j_{1} \leq \ldots \leq j_{m} \leq n} c_{j_{1} \ldots j_{m}} x_{j_{\sigma}-1(1)}^{(1)} \ldots x_{j_{\sigma}-1(m)}^{(m)} \\
& =\frac{1}{m !} \sum_{\tau \in \Sigma_{m}} \sum_{1 \leq j_{1} \leq \ldots \leq j_{m} \leq n} c_{j_{1} \ldots j_{m}} x_{j_{\tau(1)}}^{(1)} \ldots x_{j_{\tau(m)}}^{(m)} .
\end{aligned}
$$

From a probabilistic point of view, this may be restated as

$$
B\left(x^{(1)}, \ldots, x^{(m)}\right)=E\left[\sum_{1 \leq j_{1} \leq \ldots \leq j_{m} \leq n} c_{j_{1} \ldots j_{m}} x_{j_{\sigma(1)}}^{(1)} \ldots x_{j_{\sigma(m)}^{(m)}}^{(m)}\right],
$$

where expectation is taken over $\sigma \in \Sigma_{m}$ and $\Sigma_{m}$ is endowed with the equiprobability measure. In other words, $B$ is the expected value of $L_{P}$ when the order of the monomials' subindices is an equidistributed random variable. Thus, a card-shuffling procedure applied to the order of the subindices will yield a symmetrization procedure for $L_{P}$ by taking expectation. We will use the Fischer-Yates shuffle in its original version which can be found in [4]. It goes as follows. Choose a random card from an ordered deck and leave it on top. Next, choose a random card between the second and the last place and leave it in the second place, and so on. At the last step, choose between the last two cards which one will go in the penultimate place. After applying this procedure, an ordered deck will be completely shuffled, that is, any arrangement will be equally probable.

Remark 2.1. Note that at any given step, the $k$-th step say, the first $k-1$ cards (which have been previously selected) are completely random, while the last cards remain completely ordered. This special structure will be crucial in the proof of Theorem 1.3.

Next, we introduce the symmetrization procedure arising from the Fischer-Yates shuffle. For every $1 \leq k \leq m-1$ we let $\mathbb{P}_{k}$ be the probability distribution on $\Sigma_{m}$ associated to performing the first $k$ steps of the shuffling algorithm. We define the $k$-th shuffle $S_{k}$ of an $m$-form $L:\left(\mathbb{C}^{n}\right)^{m} \rightarrow \mathbb{C}$ by

$$
S_{k} L\left(x^{(1)}, \ldots, x^{(m)}\right)=E\left[\sum_{i_{1}, \ldots, i_{m}=1}^{n} c_{i_{1} \ldots i_{m}} x_{i_{\sigma(1)}}^{(1)} \ldots x_{i_{\sigma(m)}^{(m)}}^{(m)}\right],
$$

where $\sigma \sim \mathbb{P}_{k}$.

In particular, from $(3)$ and the fact that the $(m-1)$-th step of the shuffle achieves equidistribution we have

$$
B=S_{m-1} L_{P}
$$


However, it should be noticed that the intermediate shuffles are not partial symmetrizations since we are symmetrizing the monomials' subindices rather than the variables.

In order to study the structure of $S_{k}$, we define the $k$-th shuffling step $T_{k}$ of an $m$-form $L:\left(\mathbb{C}^{n}\right)^{m} \rightarrow \mathbb{C}$ by

$$
\begin{aligned}
& T_{k} L\left(x^{(1)}, \ldots, x^{(m)}\right) \\
& \quad=\frac{1}{m-k+1} \sum_{l=k}^{m} L\left(x^{(1)}, \ldots, x^{(k-1)}, x^{(k+1)}, \ldots, x^{(l)}, x^{(k)}, x^{(l+1)}, \ldots, x^{(m)}\right) .
\end{aligned}
$$

Lemma 2.2. For every $1 \leq k \leq m-1$ we have that $S_{k}=T_{k} \ldots T_{1}$.

Proof. Since $T_{k}$ and $S_{k}$ are linear for every $1 \leq k \leq m-1$, it is enough to check that the equality holds for monomials. Fix $1 \leq i_{1}, \ldots, i_{m} \leq n$, we have to prove that

$$
S_{k}\left(x_{i_{1}}^{(1)} \ldots x_{i_{m}}^{(m)}\right)=T_{k} \ldots T_{1}\left(x_{i_{1}}^{(1)} \ldots x_{i_{m}}^{(m)}\right) .
$$

We will proceed by induction. If $k=1$, the random permutation $\sigma$ is a cycle in $\Sigma_{m}$. More precisely, using the cycle notation in $\Sigma_{m}$ we have that $\sigma$ takes the value $(l l-1 \ldots 1)$ for some $1 \leq l \leq m$ with probability $1 / m$. Therefore, we get

$$
\begin{aligned}
S_{1}\left(x_{i_{1}}^{(1)} \ldots x_{i_{m}}^{(m)}\right) & =E\left[x_{i_{\sigma(1)}}^{(1)} \ldots x_{i_{\sigma(m)}}^{(m)}\right]=\frac{1}{m} \sum_{l=1}^{m} x_{i_{l}}^{(1)} x_{i_{1}}^{(2)} \ldots x_{i_{l-1}}^{(l)} x_{i_{l+1}}^{(l+1)} \ldots x_{i_{m}}^{(m)} \\
& =\frac{1}{m} \sum_{l=1}^{m} x_{i_{1}}^{(2)} \ldots x_{i_{l-1}}^{(l)} x_{i_{l}}^{(1)} x_{i_{l+1}}^{(l+1)} \ldots x_{i_{m}}^{(m)}=T_{1}\left(x_{i_{1}}^{(1)} \ldots x_{i_{m}}^{(m)}\right) .
\end{aligned}
$$

Only the inductive step remains to be proven. Let $2 \leq k \leq m-1$ and suppose the lemma holds for $k-1$. From the definition of the Fischer-Yates shuffle we may deduce that a random permutation with law $\mathbb{P}_{k}$ can be written as the composition of two independent random permutations $\tau$ and $\sigma$ where $\sigma \sim \mathbb{P}_{k-1}$ and $\tau$ takes the value $\tau_{l}=(l l-1 \ldots k)$ for some $k \leq l \leq m$ with probability $1 /(m-k+1)$. For a fixed $\tau$, we may define new indices $j_{1}, \ldots, j_{m}$ such that $j_{k}=i_{\tau(k)}$ for every $1 \leq k \leq m$. So we obtain

$$
\begin{aligned}
S_{k}\left(x_{i_{1}}^{(1)} \ldots\right. & \left.x_{i_{m}}^{(m)}\right)=E_{\tau, \sigma}\left[x_{i_{\tau \sigma(1)}}^{(1)} \ldots x_{i_{\tau \sigma(m)}}^{(m)}\right]=E_{\tau}\left[E_{\sigma}\left[x_{j_{\sigma(1)}}^{(1)} \ldots x_{j_{\sigma(m)}}^{(m)}\right]\right] \\
& =E_{\tau}\left[S_{k-1}\left(x_{j_{1}}^{(1)} \ldots x_{j_{m}}^{(m)}\right)\right]=E_{\tau}\left[S_{k-1}\left(x_{i_{\tau(1)}}^{(1)} \ldots x_{\left.i_{\tau(m)}\right)}^{(m)}\right)\right] \\
& =\frac{1}{m-k+1} \sum_{l=k}^{m} S_{k-1}\left(x_{\left.i_{\tau_{l}(1)}^{(1)} \ldots x_{i_{l}(m)}^{(m)}\right)}^{m}\right. \\
& =\frac{1}{m-k+1} \sum_{l=k}^{m} S_{k-1}\left(x_{i_{1}}^{(1)} \ldots x_{i_{k-1}}^{(k-1)} x_{i_{l}}^{(k)} x_{i_{k}}^{(k+1)} \ldots x_{i_{l-1}}^{(l)} x_{i_{l+1}}^{(l+1)} \ldots x_{i_{m}}^{(m)}\right) \\
& =\frac{1}{m-k+1} \sum_{l=k}^{m} S_{k-1}\left(x_{i_{1}}^{(1)} \ldots x_{i_{k-1}}^{(k-1)} x_{i_{k}}^{(k+1)} \ldots x_{i_{l-1}}^{(l)} x_{i_{l}}^{(k)} x_{i_{l+1}}^{(l+1)} \ldots x_{i_{m}}^{(m)}\right) \\
& =T_{k} S_{k-1}\left(x_{i_{1}}^{(1)} \ldots x_{i_{m}}^{(m)}\right)
\end{aligned}
$$

which completes the proof. 
Following [2], we turn to study how the coefficients of the succesive shuffles of $L_{P}$ change. Let $L:\left(\mathbb{C}^{n}\right)^{m} \rightarrow \mathbb{C}$ be an $m$-linear form given by

$$
L\left(x^{(1)}, \ldots, x^{(m)}\right)=\sum_{i \in \mathcal{I}(m, n)} c_{i} x_{i_{1}}^{(1)} \ldots x_{i_{m}}^{(m)},
$$

where $\mathcal{I}(m, n)=\{1, \ldots, n\}^{m}$. We will denote its coefficients by $c_{i}(L)=c_{i}$.

Lemma 2.3. For $m, n \in \mathbb{N}, 1 \leq k \leq m-1, i \in \mathcal{I}(m, n)$ and an m-homogeneous polynomial $P: \mathbb{C}^{n} \rightarrow \mathbb{C}$ we have

$c_{i}\left(S_{k-1} L_{P}\right)=\left\{\begin{array}{ll}(m-k+1)\left(1+\sum_{u=1}^{m-k} \delta_{i_{k}, i_{k+u}}\left(\frac{1}{u+1}-\frac{1}{u}\right)\right) c_{i}\left(S_{k} L_{P}\right) & \text { if } i_{k} \leq i_{k+1} \\ 0 & \text { otherwise }\end{array}\right.$,

where $\delta$ is the Kronecker delta and we take $S_{0} L_{P}=L_{P}$.

Proof. We begin the proof by calculating the coefficents $c_{i}\left(S_{k} L_{P}\right)$ in terms of the coefficients $c_{i}\left(S_{k-1} L_{P}\right)$. Observe that for an $m$-linear form $L:\left(\mathbb{C}^{n}\right)^{m} \rightarrow \mathbb{C}$ we have

$$
\begin{aligned}
T_{k} L & \left(x^{(1)}, \ldots, x^{(m)}\right) \\
& =\frac{1}{m-k+1} \sum_{l=k}^{m} L\left(x^{(1)}, \ldots, x^{(k-1)}, x^{(k+1)}, \ldots, x^{(l)}, x^{(k)}, x^{(l+1)}, \ldots, x^{(m)}\right) \\
& =\frac{1}{m-k+1} \sum_{l=k}^{m} \sum_{i \in \mathcal{I}(m, n)} c_{i}(L) x_{i_{1}}^{(1)} \ldots x_{i_{k-1}}^{(k-1)} x_{i_{k}}^{(k+1)} \ldots x_{i_{l-1}}^{(l)} x_{i_{l}}^{(k)} x_{i_{l+1}}^{(l+1)} \ldots x_{i_{m}}^{(m)} \\
& =\sum_{i \in \mathcal{I}(m, n)} \frac{1}{m-k+1} \sum_{l=k}^{m} c_{i}(L) x_{i_{1}}^{(1)} \ldots x_{i_{k-1}}^{(k-1)} x_{i_{l}}^{(k)} x_{i_{k}}^{(k+1)} \ldots x_{i_{l-1}}^{(l)} x_{i_{l+1}}^{(l+1)} \ldots x_{i_{m}}^{(m)} \\
& =\sum_{i \in \mathcal{I}(m, n)} \frac{1}{m-k+1} \sum_{l=k}^{m} c_{\left(i_{1}, \ldots, i_{k-1}, i_{k+1}, \ldots, i_{l}, i_{k}, i_{l+1}, \ldots, i_{m}\right)}(L) x_{i_{1}}^{(1)} \ldots x_{i_{m}}^{(m)} .
\end{aligned}
$$

Therefore, since $S_{k}=T_{k} S_{k-1}$, we deduce the formula

$$
c_{i}\left(S_{k} L_{P}\right)=\frac{1}{m-k+1} \sum_{l=k}^{m} c_{\left(i_{1}, \ldots, i_{k-1}, i_{k+1}, \ldots, i_{l}, i_{k}, i_{l+1}, \ldots, i_{m}\right)}\left(S_{k-1} L_{P}\right) .
$$

By the definition of $L_{P}$ if a coefficient $c_{i}\left(L_{P}\right)$ is not zero, then the index $i$ must satisfy that $1 \leq i_{1} \leq \ldots \leq i_{m} \leq n$. We will prove inductively that for $0 \leq k \leq m-1$, if the coefficient $c_{i}\left(S_{k} L_{P}\right)$ is not zero, then the index $i$ must satisfy that $1 \leq i_{k+1} \leq$ $\ldots \leq i_{m} \leq n$.

Since $S_{0} L_{P}=L_{P}$, the case $k=0$ is already proven. Now assume the assertion holds for $0 \leq k-1 \leq m-1$ and fix $i \in \mathcal{I}(m, n)$ such that $i_{s}>i_{s+1}$ for some $k+1 \leq s \leq m-1$. Applying the inductive hypothesis we may deduce that

$$
c_{\left(i_{1}, \ldots, i_{k-1}, i_{k+1}, \ldots, i_{l}, i_{k}, i_{l+1}, \ldots, i_{m}\right)}\left(S_{k-1} L_{P}\right)=0,
$$

for every $k \leq l \leq m$. Hence, using (4) we get that $c_{i}\left(S_{k} L_{P}\right)=0$ proving the inductive step. In particular, we have shown that $c_{i}\left(S_{k-1} L_{P}\right)=0$ if $i_{k}>i_{k+1}$ as sought.

Now assume that $i_{k} \leq i_{k+1}$. If for some $k+1 \leq s \leq m-1$ we have that $i_{s}>i_{s+1}$, then by the previous argument we may deduce that $c_{i}\left(S_{k-1} L_{P}\right)=c_{i}\left(S_{k} L_{P}\right)=0$ as 
desired. Therefore, it remains to check the statement when $1 \leq i_{k} \leq \ldots \leq i_{m} \leq n$. Define $s=\sup \left\{k \leq u \leq m: i_{u}=i_{k}\right\}$ and notice that

$$
c_{\left(i_{1}, \ldots, i_{k-1}, i_{k+1}, \ldots, i_{l}, i_{k}, i_{l+1}, \ldots, i_{m}\right)}\left(S_{k-1} L_{P}\right)=\left\{\begin{array}{ll}
c_{i}\left(S_{k-1} L_{P}\right) & \text { if } k \leq l \leq s \\
0 & \text { if } s<l \leq m
\end{array} .\right.
$$

Thus, we may push (4) further to get

$$
\begin{aligned}
c_{i}\left(S_{k} L_{P}\right) & =\frac{1}{m-k+1} \sum_{l=k}^{m} c_{\left(i_{1}, \ldots, i_{k-1}, i_{k+1}, \ldots, i_{l}, i_{k}, i_{l+1}, \ldots, i_{m}\right)}\left(S_{k-1} L_{P}\right) \\
& =\frac{1}{m-k+1} \sum_{l=k}^{s} c_{i}\left(S_{k-1} L_{P}\right)=\frac{s-k+1}{m-k+1} c_{i}\left(S_{k-1} L_{P}\right) .
\end{aligned}
$$

Since $s \geq k$, we have that $s-k+1 \neq 0$. Thus, we get

$$
\begin{aligned}
c_{i}\left(S_{k-1} L_{P}\right) & =\frac{m-k+1}{s-k+1} c_{i}\left(S_{k} L_{P}\right) \\
& =(m-k+1)\left(1+\sum_{u=1}^{s-k}\left(\frac{1}{u+1}-\frac{1}{u}\right)\right) c_{i}\left(S_{k} L_{P}\right) \\
& =(m-k+1)\left(1+\sum_{u=1}^{m-k} \delta_{i_{k}, i_{k+u}}\left(\frac{1}{u+1}-\frac{1}{u}\right)\right) c_{i}\left(S_{k} L_{P}\right) .
\end{aligned}
$$

This concludes the proof.

As in [2], we will restate the previous lemma using Schur products. For $A, B \in$ $\mathbb{C}^{\mathcal{I}(m, n)}$, the Schur product $A * B$ is given by

$$
c_{i}(A * B)=c_{i}(A) c_{i}(B),
$$

where $c_{i}(\cdot)$ denotes de $i$-th entry of a matrix. By identifying an $m$-linear form with its coefficients, we may compute the product between a matrix and an $m$-form. More precisely, for $A \in \mathbb{C}^{\mathcal{I}(m, n)}$ and an $m$-linear form $L:\left(\mathbb{C}^{n}\right)^{m} \rightarrow \mathbb{C}$ we define $A * L$ : $\left(\mathbb{C}^{n}\right)^{m} \rightarrow \mathbb{C}$ by

$$
c_{i}(A * L)=c_{i}(A) c_{i}(L) .
$$

With this notation Lemma 2.3 proves the formula

$$
S_{k-1} L_{P}=R_{k} * S_{k} L_{P}
$$

where $R_{k} \in \mathbb{C}^{\mathcal{I}(m, n)}$ is given by

$$
c_{i}\left(R_{k}\right)=\left\{\begin{array}{ll}
(m-k+1)\left(1+\sum_{u=1}^{m-k} \delta_{i_{k}, i_{k+u}}\left(\frac{1}{u+1}-\frac{1}{u}\right)\right) & \text { if } i_{k} \leq i_{k+1} \\
0 & \text { otherwise }
\end{array} .\right.
$$

The matrix $R_{k} \in \mathbb{C}^{\mathcal{I}(m, n)}$ may be decomposed as sums and products of simpler matrices. For $u, v \in\{1, \ldots, m\}$, let $D^{u, v}, T^{u, v} \in \mathbb{C}^{\mathcal{I}(m, n)}$ be such that for every $i \in$ $\mathcal{I}(m, n)$ we have

$$
\begin{aligned}
& c_{i}\left(D^{u, v}\right)= \begin{cases}1 & \text { if } i_{u}=i_{v} \\
0 & \text { otherwise }\end{cases} \\
& c_{i}\left(T^{u, v}\right)= \begin{cases}1 & \text { if } i_{u} \leq i_{v} \\
0 & \text { otherwise }\end{cases}
\end{aligned}
$$


Keeping Remark 1.5 in mind, we may observe that $T^{u, v}$ bears a close ressemblance with the main triangle projection $T: \mathbb{C}^{n \times n} \rightarrow \mathbb{C}^{n \times n}$. Indeed, note that $c_{i}\left(T^{u, v}\right)=c_{i_{u}, i_{v}}(T)$ for every $i \in \mathcal{I}(m, n)$.

Lemma 2.4. For $1 \leq k \leq m-1$, we have

$$
R_{k}=(m-k+1) T^{k, k+1} *\left(1+\sum_{u=1}^{m-k} D^{k, k+u}\left(\frac{1}{u+1}-\frac{1}{u}\right)\right) .
$$

Proof. For $i \in \mathcal{I}(m, n)$, we deduce that

$$
\begin{aligned}
c_{i}((m-k+1) & \left.T^{k, k+1} *\left(1+\sum_{u=1}^{m-k} D^{k, k+u}\left(\frac{1}{u+1}-\frac{1}{u}\right)\right)\right)= \\
& =(m-k+1) c_{i}\left(T^{k, k+1}\right)\left(1+\sum_{u=1}^{m-k} c_{i}\left(D^{k, k+u}\right)\left(\frac{1}{u+1}-\frac{1}{u}\right)\right) \\
& =c_{i}\left(T^{k, k+1}\right)(m-k+1)\left(1+\sum_{u=1}^{m-k} \delta_{i_{k}, i_{k+u}}\left(\frac{1}{u+1}-\frac{1}{u}\right)\right) \\
& =c_{i}\left(R_{k}\right),
\end{aligned}
$$

which proves the statement.

\section{UPPER BOUNDS}

In this section we provide the upper bounds for Theorem 1.3. Let $\|\cdot\|$ be a norm on $\mathbb{C}^{n}$. For $A \in \mathbb{C}^{\mathcal{I}(m, n)}$, we define $\mu_{\|\cdot\|}(A)$ as the infimum of the constants $C>0$ such that for every $m$-linear form $L:\left(\mathbb{C}^{n}\right)^{m} \rightarrow \mathbb{C}$ we have

$$
\sup _{\left\|x^{(k)}\right\| \leq 1}\left|A * L\left(x^{(1)}, \ldots, x^{(m)}\right)\right| \leq C \sup _{\left\|x^{(k)}\right\| \leq 1}\left|L\left(x^{(1)}, \ldots, x^{(m)}\right)\right| .
$$

Note that $\left(\mathbb{C}^{\mathcal{I}(m, n)}, \mu_{\|\cdot\|}\right)$ is a Banach algebra.

We will use the following lemma by Defant and Schlüters.

Lemma 3.1 [2, Lemma 3.2]. For every $n, m \in \mathbb{N}$, every $u, v \in\{1, \ldots, m\}$ and every 1-unconditional norm $\|\cdot\|$ on $\mathbb{C}^{n}$

$$
\begin{aligned}
& \mu_{\|\cdot\|}\left(D^{u, v}\right)=1, \\
& \mu_{\|\cdot\|}\left(T^{u, v}\right) \leq \log _{2}(2 n) .
\end{aligned}
$$

Moreover, for every $1 \leq p<2$, there exists a constant $c=c(p)$ so that for every $n, m \in \mathbb{N}$

$$
\mu_{\|\cdot\|_{p}}\left(T^{u, v}\right) \leq c .
$$

As mentioned in Remark 1.5, the estimates for $T^{u, v}$ rely on bounds for the norm of the main triangle projection obtained by Kwapień and Pełczyński in [5] and Bennett in [1].

Corollary 3.2. For every $n, m \in \mathbb{N}$, every $1 \leq k \leq m-1$ and every 1 -unconditional norm $\|\cdot\|$ on $\mathbb{C}^{n}$ we have

$$
\mu_{\|\cdot\|}\left(R_{k}\right) \leq 2(m-k+1) \mu_{\|\cdot\|}\left(T^{k, k+1}\right) .
$$


Proof. From the last lemma we know that $\mu_{\|\cdot\|}\left(D^{u, v}\right)=1$ for every $u, v \in\{1, \ldots, m\}$. Since $\left(\mathbb{C}^{\mathcal{I}(m, n)}, \mu_{\|\cdot\|}\right)$ is a Banach algebra, we may deduce from Lemma 2.4 that

$$
\begin{aligned}
\mu_{\|\cdot\|}\left(R_{k}\right) & =\mu_{\|\cdot\|}\left((m-k+1) T^{k, k+1} *\left(1+\sum_{u=1}^{m-k} D^{k, k+u}\left(\frac{1}{u+1}-\frac{1}{u}\right)\right)\right) \\
& \left.\leq(m-k+1) \mu_{\|\cdot\|}\left(T^{k, k+1}\right)\left(1+\sum_{u=1}^{m-k} \mu_{\|\cdot\|}\left(D^{k, k+u}\right) \mid \frac{1}{u+1}-\frac{1}{u}\right)\right) \\
& \leq(m-k+1)\left(1+\sum_{u=1}^{\infty}\left(\frac{1}{u}-\frac{1}{u+1}\right)\right) \mu_{\|\cdot\|}\left(T^{k, k+1}\right) \\
& =2(m-k+1) \mu_{\|\cdot\|}\left(T^{k, k+1}\right),
\end{aligned}
$$

as required.

We are ready to prove the upper bounds for Theorem 1.3.

Theorem 3.3. There exists a universal constant $c_{1} \geq 1$ such that

$$
C(m, n) \leq c_{1}^{m} m^{m}(\log n)^{m-1} .
$$

Moreover, for $1 \leq p<2$, there is a constant $c_{2}=c_{2}(p) \geq 1$ for which

$$
C_{p}(m, n) \leq c_{2}^{m} m^{m}
$$

Proof. Using (5), the definition of $\mu_{\|\cdot\|}$ and the previous corollary we get

$$
\begin{aligned}
\sup _{\left\|x^{(k)}\right\| \leq 1} \mid S_{k-1} L_{P}\left(x^{(1)}\right. & \left., \ldots, x^{(m)}\right)\left|=\sup _{\left\|x^{(k)}\right\| \leq 1}\right| R_{k} * S_{k} L_{P}\left(x^{(1)}, \ldots, x^{(m)}\right) \mid \\
& \leq \mu_{\|\cdot\|}\left(R_{k}\right) \sup _{\left\|x^{(k)}\right\| \leq 1}\left|S_{k} L_{P}\left(x^{(1)}, \ldots, x^{(m)}\right)\right| \\
& \leq 2(m-k+1) \mu_{\|\cdot\|}\left(T^{k, k+1}\right) \sup _{\left\|x^{(k)}\right\| \leq 1}\left|S_{k} L_{P}\left(x^{(1)}, \ldots, x^{(m)}\right)\right|,
\end{aligned}
$$

for every $1 \leq k \leq m-1$. Taking $\mu=\sup _{1 \leq k \leq m-1} \mu_{\|\cdot\|}\left(T^{k, k+1}\right)$ and linking the previous inequalities together, we deduce that

$$
\begin{aligned}
\sup _{\left\|x^{(k)}\right\| \leq 1}\left|L_{P}\left(x^{(1)}, \ldots, x^{(m)}\right)\right| & \leq 2 m \mu \sup _{\left\|x^{(k)}\right\| \leq 1}\left|S_{1} L_{P}\left(x^{(1)}, \ldots, x^{(m)}\right)\right| \\
& \leq 2^{2} m(m-1) \mu^{2} \sup _{\left\|x^{(k)}\right\| \leq 1}\left|S_{2} L_{P}\left(x^{(1)}, \ldots, x^{(m)}\right)\right| \\
& \leq \ldots \leq 2^{m-1} m ! \mu^{m-1} \sup _{\left\|x^{(k)}\right\| \leq 1}\left|S_{m-1} L_{P}\left(x^{(1)}, \ldots, x^{(m)}\right)\right| .
\end{aligned}
$$

Using the identity $S_{m-1} L_{P}=B$ and applying (1), we obtain

$$
\begin{aligned}
\sup _{\left\|x^{(k)}\right\| \leq 1}\left|L_{P}\left(x^{(1)}, \ldots, x^{(m)}\right)\right| & \leq 2^{m-1} m ! \mu^{m-1} \sup _{\left\|x^{(k)}\right\| \leq 1}\left|B\left(x^{(1)}, \ldots, x^{(m)}\right)\right| \\
& \leq 2^{m-1} e^{m} m ! \mu^{m-1} \sup _{\|x\| \leq 1}|P(x)| .
\end{aligned}
$$

The theorem follows by applying Stirling's formula to estimate $m$ ! and Lemma 3.1 to estimate $\mu$. 


\section{LOWER BOUnds}

Firstly, we provide a lower bound for $C_{p}(m, n)$.

Lemma 4.1. For every $n \geq m$ and every $1 \leq p<2$, we have that $C_{p}(m, n) \geq m^{\frac{m}{p}}$.

Proof. Let $P: \mathbb{C}^{m} \rightarrow \mathbb{C}$ be the $m$-homogeneous polynomial defined by

$$
P(x)=x_{1} \ldots x_{m} .
$$

So, its associated $m$-linear form $L_{P}:\left(\mathbb{C}^{m}\right)^{m} \rightarrow \mathbb{C}$ is given by

$$
L_{P}\left(x^{(1)}, \ldots, x^{(m)}\right)=x_{1}^{(1)} \ldots x_{m}^{(m)} .
$$

Observe that

$$
\sup _{\left\|x^{(k)}\right\|_{p} \leq 1}\left|L_{P}\left(x^{(1)}, \ldots, x^{(m)}\right)\right|=\sup _{\left\|x^{(k)}\right\|_{p} \leq 1}\left|x_{1}^{(1)} \ldots x_{m}^{(m)}\right|=1 .
$$

where equality is achieved by taking $x^{(i)}$ to be the $i$-th canonical vector of $\ell_{p}^{m}$.

On the other hand, a straightforward computation using Lagrange multipliers gives

$$
\sup _{\|x\|_{p} \leq 1}|P(x)|=\left|P\left(m^{-\frac{1}{p}}(1, \ldots, 1)\right)\right|=m^{-\frac{m}{p}} .
$$

Applying (6) and (7) together with the definition of $C_{p}(m, n)$ we get

$$
1=\sup _{\left\|x^{(k)}\right\|_{p} \leq 1}\left|L_{P}\left(x^{(1)}, \ldots, x^{(m)}\right)\right| \leq C_{p}(m, n) \sup _{\|x\|_{p} \leq 1}|P(x)|=m^{-\frac{m}{p}} C_{p}(m, n),
$$

as desired.

Secondly, we estimate $C(m, n)$ from below. In order to do this we will need the following special case of a theorem proved by Pełczyński.

Theorem 4.2 [8, Theorem 1]. For a finite index set $J$, let $\left(a_{j}\right)_{j \in J}$ and $\left(b_{j}\right)_{j \in J}$ be sequences of characters on compact abelian groups $S$ and $T$ respectively. Suppose there are constants $c_{1}, c_{2}>0$ such that

$$
\frac{1}{c_{1}}\left\|\sum_{j \in J} \alpha_{j} a_{j}\right\|_{C(S)} \leq\left\|\sum_{j \in J} \alpha_{j} b_{j}\right\|_{C(T)} \leq c_{2}\left\|\sum_{j \in J} \alpha_{j} a_{j}\right\|_{C(S)}
$$

for every sequence of scalars $\left(\alpha_{j}\right)_{j \in J} \subseteq \mathbb{C}$. Then, for every Banach space $E$ and every sequence of vectors $\left(v_{j}\right)_{j \in J} \subseteq E$ we have

$$
\frac{1}{c_{1} c_{2}} \int_{S}\left\|\sum_{j \in J} v_{j} a_{j}(s)\right\|_{E} d s \leq \int_{T}\left\|\sum_{j \in J} v_{j} b_{j}(t)\right\|_{E} d t \leq c_{1} c_{2} \int_{S}\left\|\sum_{j \in J} v_{j} a_{j}(s)\right\|_{E} d s .
$$

We are ready to provide the lower bound for $C(m, n)$ stated in Theorem 1.3.

Lemma 4.3. For $n, m \in \mathbb{N}$ such that $\log \left(\frac{2 n}{m}\right) \geq \pi$, we have

$$
C(m, n) \geq\left(\frac{\log \left(\frac{2 n}{m}\right)-\pi}{\pi}\right)^{m / 2}
$$


Proof. Consider the norm $\|\cdot\|_{\infty}$ on $\mathbb{C}^{n}$. Since $P(x)=L_{P}(x, \ldots, x)$, we deduce that

$$
\sup _{\|x\|_{\infty} \leq 1}|P(x)| \leq \sup _{\left\|x^{(k)}\right\|_{\infty} \leq 1}\left|L_{P}\left(x^{(1)}, \ldots, x^{(m)}\right)\right| \leq C(m, n) \sup _{\|x\|_{\infty} \leq 1}|P(x)|,
$$

for every $m$-homogeneous polynomial $P: \mathbb{C}^{n} \rightarrow \mathbb{C}$. Equivalently, by the maximum modulus principle we get

$$
\sup _{x \in \mathbb{T}^{n}}|P(x)| \leq \sup _{x^{(k)} \in \mathbb{T}^{n}}\left|L_{P}\left(x^{(1)}, \ldots, x^{(m)}\right)\right| \leq C(m, n) \sup _{x \in \mathbb{T}^{n}}|P(x)|,
$$

where $\mathbb{T}=\{z \in \mathbb{C}:|z|=1\}$.

Thus, the conditions of Pełczyński's theorem are satisfied. Indeed, denote the compact abelian groups $\mathbb{T}^{n}$ and $\left(\mathbb{T}^{n}\right)^{m}$ by $S$ and $T$ respectively and consider the index set $J=\left\{j \in \mathcal{I}(m, n): 1 \leq j_{1} \leq \ldots \leq j_{m} \leq n\right\}$. For every $j \in J$, define the characters $a_{j}: S \rightarrow \mathbb{T}$ and $b_{j}: T \rightarrow \mathbb{T}$ by

$$
a_{j}(x)=x_{j_{1}} \ldots x_{j_{m}} \quad \text { and } \quad b_{j}\left(x^{(1)}, \ldots, x^{(m)}\right)=x_{j_{1}}^{(1)} \ldots x_{j_{m}}^{(m)} .
$$

If we restate (10) with this notation we get (8), with $c_{1}=1$ and $c_{2}=C(m, n)$. Therefore, we deduce from Pełczyński's theorem that

$$
\begin{aligned}
\frac{1}{C(m, n)} \int_{\mathbb{T}^{n}}\left\|\sum_{j \in J} v_{j} x_{j_{1}} \ldots x_{j_{m}}\right\|_{E} d x & \leq \int_{\mathbb{T}^{n}} \ldots \int_{\mathbb{T}^{n}}\left\|\sum_{j \in J} v_{j} x_{j_{1}}^{(1)} \ldots x_{j_{m}}^{(m)}\right\|_{E} d x^{(1)} \ldots d x^{(m)} \\
& \leq C(m, n) \int_{\mathbb{T}^{n}}\left\|\sum_{j \in J} v_{j} x_{j_{1}} \ldots x_{j_{m}}\right\|_{E} d x .
\end{aligned}
$$

for every Banach space $E$ and every sequence of vectors $\left(v_{j}\right)_{j \in J} \subseteq E$. Choosing the space $E$ and the vectors $\left(v_{j}\right)_{j \in J} \subseteq E$ adequately will yield the estimate we seek.

We will build upon an example provided by Bourgain (unpublished) and included in a paper by McConnell and Taqqu [7, Example 4.1] (see also [6, Section 6.9]). Consider the Banach space $F=\mathcal{L}\left(\ell_{2}\right)$. For every $1 \leq i \neq j \leq n$, define vectors $v_{i j} \in F$ by

$$
v_{i j}=\frac{1}{i-j} e_{i} \otimes e_{j}+\frac{1}{j-i} e_{j} \otimes e_{i} .
$$

Using complex Steinhaus variables instead of Bernoulli random variables and proceeding as in [6] we get

$$
\begin{gathered}
\int_{\mathbb{T}^{n}}\left\|\sum_{1 \leq i<j \leq n} v_{i j} x_{i} x_{j}\right\| d x \leq \pi \quad \text { and } \\
\int_{\mathbb{T}^{n}} \int_{\mathbb{T}^{n}}\left\|\sum_{1 \leq i<j \leq n} v_{i j} x_{i}^{(1)} x_{j}^{(2)}\right\| d x^{(1)} d x^{(2)} \geq \log n-\pi .
\end{gathered}
$$

Note that by the previous estimations we obtain the desired result for $m=2$ since we have

$$
\begin{array}{r}
\log n-\pi \leq \int_{\mathbb{T}^{n}} \int_{\mathbb{T}^{n}}\left\|\sum_{1 \leq i<j \leq n} v_{i j} x_{i}^{(1)} x_{j}^{(2)}\right\| d x^{(1)} d x^{(2)} \\
\leq C(2, n) \int_{\mathbb{T}^{n}}\left\|\sum_{1 \leq i<j \leq n} v_{i j} x_{i} x_{j}\right\| d x \leq C(2, n) \pi .
\end{array}
$$


Moreover, this together with Theorem 3.3 shows that the asymptotic behaviour of $C(2, n)$ is logarithmic.

To conclude our argument it remains to extend this 2-variable example to $m$ variables. Assume $m$ is even and let $E=\bigotimes_{k=1}^{m / 2} F$ be the projective tensor product of $m / 2$ copies of $F$. Consider the $m$-homogeneous vector-valued polynomial $P: \mathbb{C}^{n} \rightarrow E$ defined by

$$
P(x)=\sum_{\substack{\frac{2 n}{m}(k-1)<j_{2 k-1}<j_{2 k} \leq \frac{2 n}{m} k \\ 1 \leq k \leq \frac{m}{2}}} v_{j} x_{j}, \quad \text { where } v_{j}=v_{j_{1} j_{2}} \otimes v_{j_{3} j_{4}} \otimes \ldots \otimes v_{j_{m-1} j_{m}} .
$$

Notice that

Applying (12) we get

$$
P(x)=\otimes_{k=1}^{m / 2} \sum_{\frac{2 n}{m}(k-1)<j_{2 k-1}<j_{2 k} \leq \frac{2 n}{m} k} v_{j_{2 k-1} j_{2 k}} x_{j_{2 k-1}} x_{j_{2 k}} .
$$

$$
\begin{aligned}
\int_{\mathbb{T}^{n}}\|P(x)\| d x & =\int_{\mathbb{T}^{n}} \prod_{k=1}^{m / 2}\left\|\sum_{\frac{2 n}{m}(k-1)<j_{2 k-1}<j_{2 k} \leq \frac{2 n}{m} k} v_{j_{2 k-1} j_{2 k}} x_{j_{2 k-1}} x_{j_{2 k}}\right\| d x \\
& =\prod_{k=1}^{m / 2} \int_{\mathbb{T}^{n}}\left\|\sum_{\frac{2 n}{m}(k-1)<j_{2 k-1}<j_{2 k} \leq \frac{2 n}{m} k} v_{j_{2 k-1} j_{2 k}} x_{j_{2 k-1}} x_{j_{2 k}}\right\| d x \\
& \leq \prod_{k=1}^{m / 2} \pi=\pi^{m / 2} .
\end{aligned}
$$

On the other hand, from (13) we deduce

$$
\begin{aligned}
\int_{\mathbb{T}^{n}} \ldots \int_{\mathbb{T}^{n}} & \left\|L_{P}\left(x^{(1)}, \ldots, x^{(m)}\right)\right\| d x^{(1)} \ldots d x^{(m)}= \\
& =\int_{\mathbb{T}^{n}} \ldots \int_{\mathbb{T}^{n}} \prod_{k=1}^{m / 2}\left\|\sum_{\frac{2 n}{m}(k-1)<j_{2 k-1}<j_{2 k} \leq \frac{2 n}{m} k} v_{j_{2 k-1} j_{2 k}} x_{j_{2 k-1}}^{(2 k-1)} x_{j_{2 k}}^{(2 k)}\right\| d x^{(1)} \ldots d x^{(m)} \\
& =\prod_{k=1}^{m / 2} \int_{\mathbb{T}^{n}} \int_{\mathbb{T}^{n}}\left\|\sum_{\frac{2 n}{m}(k-1)<j_{2 k-1}<j_{2 k} \leq \frac{2 n}{m} k} v_{j_{2 k-1} j_{2 k}} x_{j_{2 k-1}}^{(2 k-1)} x_{j_{2 k}}^{(2 k)}\right\| d x^{(2 k-1)} d x^{(2 k)} \\
& \geq \prod_{k=1}^{m / 2}\left(\log \left(\frac{2 n}{m}\right)-\pi\right)=\left(\log \left(\frac{2 n}{m}\right)-\pi\right)^{m / 2} .
\end{aligned}
$$

Finally, using (11) together with these estimates we obtain

$$
C(n, m) \geq\left(\frac{\log \left(\frac{2 n}{m}\right)-\pi}{\pi}\right)^{m / 2}
$$

as desired.

Note that Lemmas 4.1 and 4.3 together with Theorem 3.3 prove Theorem 1.3.

Remark 4.4. Tracing back the argument to obtain (13), we find that Bourgain's example is based on a lower estimate of the main triangle projection's norm on $\mathcal{L}\left(\ell_{2}\right)$. 
In other words, the lower bound for $C(m, n)$ was obtained by studying the behaviour of the main triangle projection as mentioned in Remark 1.5. Although $C(m, n)$ and $C_{p}(m, n)$ were not completely characterized, it seems that the main triangle projection plays a crucial role in determining their asymptotic behaviour.

\section{ACKNOWLEDGEMENTS}

I thank my supervisor Daniel Carando for his guidance and fruitful discussions and Sunke Schlüters for his helpful comments.

\section{REFERENCES}

[1] Bennett, G. "Unconditional convergence and almost everywhere convergence". In: Zeitschrift für Wahrscheinlichkeitstheorie und Verwandte Gebiete 34.2 (1976), pp. 135-155.

[2] Defant, A. and Schlüters, S. "Non-symmetric polarization". In: Journal of Mathematical Analysis and Applications 445.2 (2017), pp. 1291-1299.

[3] Dineen, S. Complex analysis on infinite dimensional spaces. London: Springer-Verlag, 1999.

[4] Fisher, R. A. and Yates, F. Statistical tables for biological, agricultural and medical research. Edinburgh: Oliver and Boyd, 1938, p. 285.

[5] Kwapień, S. and Pełczyński, A. "The main triangle projection in matrix spaces and its applications". eng. In: Studia Mathematica 34.1 (1970), pp. 43-67.

[6] Kwapien, S. and Woyczynski, W. Random series and stochastic integrals: single and multiple. Birkhäuser Basel, 1992.

[7] McConnell, T. R. and Taqqu, M. S. "Decoupling of Banach-valued multilinear forms in independent symmetric Banach-valued random variables". In: Probability Theory and Related Fields 75.4 (1987), pp. 499-507.

[8] Pełczyński, A. "Commensurate Sequences of Characters". In: Proceedings of the American Mathematical Society 104.2 (1988), pp. 525-531.

Departamento de Matemática - Pab i, Facultad de Cs. Exactas y Naturales, Universidad de Buenos Aires, (1428) Buenos Aires, Argentina, and COniCET-IMAS 